synthesis by a COX2-specific small interfering RNA also reduced medulloblastoma proliferation. Experiments in mice carrying human medulloblastoma xenografts showed that treatment with diclofenac or celecoxib reduced tumor growth and microvessel density compared with untreated animals. Levels of the apoptosis marker caspase-3 were elevated in tumors from treated mice.

The authors suggest that therapies such as COX inhibitors that target prostanoid production might have therapeutic benefit against human medulloblastoma.

Original article Baryawno N et al. (2008) Tumor-growthpromoting cyclooxygenase-2 prostaglandin E2 pathway provides medulloblastoma therapeutic targets. Neuro Oncol 10: 661-674

\section{Lewy body pathology is seen in cutaneous nerves and can be detected in skin biopsy samples}

Involvement of the peripheral autonomic nervous system (PANS) is an important feature of Lewy body diseases (LBDs) such as Parkinson disease (PD). The PANS structures in which Lewy body (LB) pathology manifests - the sympathetic ganglia and adrenal glands-are not appropriate sites for diagnostic biopsy. Ikemura et al. have shown that LB-related pathology manifests in the cutaneous nerves in patients with proven LBD.

First, the authors assessed LB pathology in skin biopsy samples taken prospectively at autopsy from 279 consecutive patients and compared the incidence with that in the CNS and adrenal glands in the same patients. A total of $20(23.5 \%)$ of the 85 patients who had CNS LB pathology also had LBs in the dermal nerve fibers. There was no evidence of LB pathology in the skin in any of the 194 individuals who did not have CNS LB pathology; therefore, the specificity of dermal LB pathology for detection of LBD was $100 \%$.

Second, the authors retrospectively assessed LB pathology in 142 autopsied patients with subclinical LBD. In this cohort, the sensitivity of skin LB pathology for CNS LBD was only 20\%; however, in patients who had PD with or without dementia or had dementia with LBs, the sensitivity rose to $70 \%$ and $40.4 \%$, respectively. The presence of dermal LB pathology was associated with decreased activities of daily living score.

The authors conclude that skin biopsy might have a diagnostic application in patients with PD with or without dementia.

Original article Ikemura $\mathrm{M}$ et al. (2008) Lewy body pathology involves cutaneous nerves. J Neuropathol Exp Neurol 67: 945-953

\section{A miniature implantable neurostimulator can treat hemicrania continua}

Occipital nerve stimulation (ONS) is an effective treatment for primary headache. However, current methods of ONS involve electrodes placed across the occipital nerve that are connected to a battery pack situated in the chest wall or abdomen; migration of the connecting leads is, therefore, a common complication that can require repeat surgery. Burns et al. used a self-contained miniature implantable neurostimulator, called a bion, to deliver ONS to six patients with hemicrania continua, a rare unilateral form of primary headache.

The bion, a $27 \times 3.3 \mathrm{~mm}$ telemetrically programmable neurostimulator with an integrated electrode and battery rechargeable with an external device, was implanted close to the occipital nerve ipsilateral to the headache site. The six patients were aged 37-64 years with a median 14-year history of hemicrania continua. The implant was activated for 3 months from the day of activation, switched off for the fourth month, and then reactivated for long-term follow-up (range 6-21 months).

Four of the six patients reported substantial improvement (80-95\%) in their headache symptoms, and one patient reported a $30 \%$ improvement. The sixth patient reported a $20 \%$ worsening in his headache at 6 months' follow-up. Although the authors recognize the possibility of a placebo effect in the successful outcomes, the delays (of days to weeks) experienced by some patients between activation or deactivation of the implant and subsequent changes in headache symptoms make a placebo effect less probable.

Original article Burns B et al. (2008) Treatment of hemicrania continua by occipital nerve stimulation with a bion device: long-term follow-up of a crossover study. Lancet Neurol 7: 1001-1012 